\title{
Score Level Fusion of Multimodal Biometrics based on Entropy Function
}

\author{
Sheikh Moeen UI Haque \\ Jamia Hamdard \\ Jamia Hamdard University \\ New Delhi-62, India
}

\author{
Moin Uddin \\ Jamia Hamdard \\ Jamia Hamdard University \\ New Delhi-62, India
}

\author{
Jyotsana Grover, PhD \\ Jamia Hamdard \\ Jamia Hamdard University \\ New Delhi-62, India
}

\begin{abstract}
This paper presents the score level fusion of multimodal biometrics using Hanman-Anirban entropy function. Entropy function captures the uncertainty in the scores. The experimental results ascertain that Entropy based score level fusion outperforms over existing methods of score level fusion such as t-norms, sum and max. We have validated our claim on finger-knuckle-print (FKP) dataset consisting of left index, left middle, right index and right middle FKP. The features of FKPs are extracted using the Gabor Wavelet. The implementation is done using MATLAB and the performance of the proposed technique is evaluated using Receiver Operating characteristics (ROC) curve. The proposed score level fusion approach achieves significant improvement in the performance over the individual FKP. We obtain Genuine acceptance rate of $99 \%$ with FAR of $0.001 \%$.
\end{abstract}

\section{General Terms}

Multimodal Biometrics, Entropy, Authentication.

\section{Keywords}

Finger Knuckle Print, Score Level Fusion, t-norms, Gabor Wavelet, Entropy Function, Biometric-Authentication.

\section{INTRODUCTION}

One of the major advantages of using biometrics as an authentication system is that it authorizes individuals based on their physiological and/or behavioral triats that are unique and inherent to everyone and cannot be lost or handed over to other individuals like tradiotnal methods of authentication such as passwords, tokens etc [1]. In the real world applications, unimodal biometric system which uses single triat often suffers from various drawbacks such as noisy data, quality of the sample, its orientation/rotation and distortion, intra class variation, recognition accuracy, non versality, spoofing etc. Recognition based on multiple biometrics can overcome these problems and is an effective way to improve the performance by utilizing evidences from different biometric triats which can be integrated at the Sensor level ( integrating the multispectral images), Feature level (integrating the features of different biometrics), Score level (combining the imposter and genuine score) and Decision level (combining the decisions). The information content is rich in the first two levels but fusion at this level may not be compatible. The fused feature space is of large dimensionality may bring irrelevant and the redundant information. Score level fusion is fairly popular and simple because it contains the richest information about the input pattern and contains ample information to differentiate between genuine and imposter scores. Fusion at decision level is considered inferior because it has less information content than Score level [2]. In Biometric system fusion at score level is considered to be dominant and is the most commonly adopted method of fusion for researchers. In score level fusion technique we have to identify pattern only in two classes: genuine (accept) and imposter (reject). Different types of classifiers have been used to combine the matching scores and arrive at a decision.

\subsection{Literature Review}

Luca et al. [3] used fingerprint and face to be fused at the score level by using Mean rule, product rule and Bayesian rule fusion techniques with FAR of $0 \%$ and FRR of $0.6 \%$ to $1.6 \%$.. PCA and LDA are used for the feature extraction and classification. Kartik et al. [4] combined speech and signature by using sum rule as fusion technique after the min max normalization is applied. Euclidean distance is used as the classification technique with $81.25 \%$ accuracy performance rate. Rodriguez et al. [5] used signature with iris by using sum rule and product rule as the fusion techniques. Neural Network is used as the classification technique with EER below than 2.0\%. Fierrez-Aguilar and Ortega-Garcia [6] , fusing face, minutiae-based finger and online signature at matching score level. This fusion approach obtained Equal Error Rate (EER) of 0.5. Viriri and Tapamo [7] introduced a multimodal approach including Iris and signature biometrics at score level fusion. This system achieves false reject rate (FRR) $0.008 \%$ on a false accept rate (FAR) of $0.01 \%$. 2. Kazi and Rody [8] presented a multimodal biometric system using face and signature with score level fusion. The results showed that face and signature based bimodal biometric system can improve the accuracy rate about $10 \%$, higher than single face/signature based biometric system. Fahmy et al.[9] Score level fusion of iris and fingerprint biometrics is carried out via Support Vector Machine (SVM). X. Zhou and B. Bhanu [10] proposed fusion of side face and gait at matching score level. They got side face features (EFSI) and gait features (GEI) from video, then fused at matching level using three strategies: Sum, Product and Max. Alford and Hansen [11], a fusion of face and periocular biometrics at the score level based on Genetic and evolutionary computations (GEC) was achieved. Their work showed that better accuracies could be reached using this technique. Ribaric and Fratric [12] acquired images containing both fingerprints and palm prints and then used the extracted eigenpalm and eigenfinger features to perform matching score level fusion. Snelick et al. [13] demonstrated that the multimodal fingerprint and face biometric system, which combines the two biometric traits at the matching score level, was significantly more accurate than any individual biometric systems.

Nandkumar et al. [14] implemented score level fusion of face, fingerprint, iris and speech modalities using the likelihood ratio based on estimation of matching score densities. The distributions of genuine and imposter match scores are modeled as a finite Gaussian mixture model in order to produce robust results. Mingxing et al. [15], the performance of sum rule-based score level fusion and SVM 
based score level fusion is performed. Nanni et al. [16] used the parameters of a finite Gaussian mixture model for modeling the genuine and impostor score densities during the fusion step. Toh et al. [17], score level fusion is also addressed from the point of view of error minimization. Here the target performance was directly optimized with respect to fusion classifier design. Park and Park [18] implemented Score level fusion for iris recognition achieved by using HD (Hamming distance) produced by a Gabor filter. Kittler et al. [19] have developed a theoretical framework for consolidating the evidence obtained from multiple classifiers using schemes like the sum rule, product rule, max rule, min rule, median rule and majority voting. Kumar et al.[20] combines iris and palmprint, the score level fusion rules (sum, product, exponential sum, tan-hyperbolic sum) are adapted using particle swarm optimization. Hanmandlu et al. [21] proposed the score level fusion of multimodal biometrics using t-norms (Hamacher (Hm), Yager (Yg), Frank (Fk), Einstein product (Ep), etc.) on three biometric traits (index, middle fingers and palmprint) to confirm the effectiveness of score level fusion.

\subsection{Motivation}

The entropy function has been used in literature for feature extraction so we will now employ this for score level fusion. Till now no one has addressed the uncertainty in scores. Score level fusion using entropy function has an edge over conventional Score level fusion, as it allows representing the information/uncertainty contained in the scores and further it allows the parameters of Entropy function to be tuned in order to tackle the uncertainty. The main motivation of our research was to achieve better performance out of Entropy based score level fusion as compared to conventional score level fusion methods like Frank t-norm, Hamacher t-norm, Sum and Max simply by tuning the parameters of Entropy based score level fusion.

\subsection{Organization Of Paper}

The organization of this paper is as follows. Section 2 describes the different entropy functions and their formulation that will be used in our experiment. Section 3 describes the triangular norms and we will be using t-norms in Hanman and Anirban entropy functions. A classifier based on the entropy function is developed using t-norms in Section 4. Results of Score level fusion using entropy function are presented in section 5. The conclusions are given in Section 6.

\section{DIFFERENT TYPES OF ENTROPY FORMULATION}

Entropy is a measure of uncertainty or disorder in a system. The uncertainty in information source is represented by different entropy functions. Shannon's entropy [22] is the spearheading work on the information measure. This entropy function is defined as a measure of uncertainty in the probability distribution and is given by the following formula:

$H^{S h}=-\sum p \log p$

where $\mathrm{p}$ is the probability, $\log \mathrm{p}$ is the logarithmic gain function and $\sum p=1$.

Renyi [23] has expanded the definition of Shannon's entropy by incorporating a parameter $\alpha$ called the power of probability which controls the shape of probability distribution. The Renyi entropy of order $\alpha$ is of the form:

$H^{R N}=\frac{\log \sum P^{\alpha}}{1-\alpha} \quad$ where $\alpha>0, \alpha \neq 1$ where $\alpha$ tends to 1, Renyi's entropy matches with Shannon's entropy and is considered special case of Shannon's entropy.

The Tsallis [25] entropy that satisfies the non-extensive property is defined as:

$H^{T S}=\frac{1-\sum P^{\alpha}}{\alpha-1}$

Pal \& Pal [26] later supplanted the logarithmic gain and touched the exponential gain function as a remedy to the shortcomings of the logarithmic gain function of Shannon with the entropy function as:

$H^{P P}=\sum \mathrm{pe}^{1-p}$

Hanmandlu and Das [27] have generalized the exponential gain in (4) with the cubic polynomial of $p$ in the exponential function as the gain, given by

$\mathrm{H}(\mathrm{p})=\sum \mathrm{pe}^{-\left(a \mathrm{p}^{3}+b \mathrm{p}^{2}+c \mathrm{p}+\mathrm{d}\right)}$

where a,b,c, and d are real valued parameters. This entropy function behaves like Pal \& Pal's entropy function with the choice of parameters in (5) as $a=0, b=0, c=1$ and $d=-1$. The parameters of this entropy function provides control on the information gain and proper tuning of these parameters by way of optimisation leads to the correct measure of uncertainty.

Mamta and Hanmandlu [28] have now modified the exponentia 1 gain as well as information source variable

$\mathrm{H}=\sum \mathrm{p}^{\gamma} \mathrm{e}^{-\left(\mathrm{ap}{ }^{\alpha}+\mathrm{b}\right)^{\beta}}$

The shape of the probability distribution can be changed by selecting the real valued parameters $\alpha, \beta, \mathrm{a} \& \mathrm{~b}$ where as $\gamma$ modifies the information source.

\section{TRINGULAR NORMS}

Binary functions like Triangular norms (t-norms) and tconorms (s-norm) very efficiently satisfy the necessary requirements of Conjunction and Disjunction operators respectively [29]. The minimum operator is of the triangular norms and the maximum operator is of s-norm. These t-norms $\mathrm{T}\left(\mathrm{P}_{1}, \mathrm{P}_{2}\right)$, and t-conorms $\mathrm{S}\left(\mathrm{P}_{1}, \mathrm{P}_{2}\right)$, are two place functions that map the unit square into the unit interval, i.e., $\mathrm{T}\left(\mathrm{P}_{1}, \mathrm{P}_{2}\right)$ : $[0,1] \times[0,1] \rightarrow[0,1]$ and $\mathrm{S}\left(\mathrm{P}_{1}, \mathrm{P}_{2}\right):[0,1] \times[0,1] \rightarrow[0,1]$

A t-norm is a function $\mathrm{T}:[0,1] \times[0,1] \rightarrow[0,1]$ which satisfies the following properties:

(a) Commutativity: $\mathrm{T}\left(\mathrm{P}_{1}, \mathrm{P}_{2}\right)=\mathrm{T}\left(\mathrm{P}_{2}, \mathrm{P}_{1}\right)$

(b) Monotonicity: $\mathrm{T}\left(\mathrm{P}_{1}, \mathrm{P}_{2}\right) \leq \mathrm{T}\left(\mathrm{P}_{3}, \mathrm{P}_{4}\right)$ if $\mathrm{P}_{1} \leq \mathrm{P}_{2}$ and $\mathrm{P}_{3}<\mathrm{P}_{4}$

(c) Associativity: $\mathrm{T}\left(\mathrm{P}_{1}, T\left(\mathrm{P}_{2}, \mathrm{P}_{3}\right)\right)=\mathrm{T}\left(\mathrm{T}\left(\mathrm{P}_{1}, \mathrm{P}_{2}\right), \mathrm{P}_{3}\right)$

(d) The number 1 acts as identity element: $T\left(P_{1}, 1\right)=P_{1}$

With the T-norms there are the S-norms which is a dual to tnorm which satisfies the following properties:

(a) Commutativity: $\mathrm{S}\left(\mathrm{P}_{1}, \mathrm{P}_{2}\right)=\mathrm{S}\left(\mathrm{P}_{2}, \mathrm{P}_{1}\right)$

(b) Monotonicity: $\mathrm{S}\left(\mathrm{P}_{1}, \mathrm{P}_{2}\right) \leq \mathrm{S}\left(\mathrm{P}_{2}, \mathrm{P}_{1}\right)$ if $\mathrm{P}_{1} \leq \mathrm{P}_{2}$ and $\mathrm{P}_{3}<\mathrm{P}_{4}$

(c) Associativity: $\mathrm{S}\left(\mathrm{P}_{1}, \mathrm{~S}\left(\mathrm{P}_{2}, \mathrm{P}_{3}\right)\right)=\mathrm{S}\left(\mathrm{S}\left(\mathrm{P}_{1}, \mathrm{P}_{2}\right), \mathrm{P}_{3}\right)$

(d) The number 0 acts as identity element: $\mathrm{S}\left(\mathrm{P}_{1}, 0\right)=\mathrm{P}_{1}$ 
As t-norms are associative, thus t-norms do not require assumption of evidential modalities to be fused and also fusion of modalities can be done irrespective of their order. Their corresponding boundary conditions, i.e., the evaluation of the t-conorms and t-norms at the extremes of the $[0,1]$ interval, satisfy the truth tables of the logical OR and AND operators. These norms stretch the maximum and the minimum of OR and AND operators respectively thus providing a better representation of sum (t-conorm) and product (t-norm) of two variables. They are related by the DeMorgan duality, which states that if $\mathrm{N}\left(\mathrm{P}_{1}\right)$ is a negation operator, then the t-conorm $\mathrm{S}\left(\mathrm{P}_{1}, \mathrm{P}_{2}\right)$ can be defined as $\mathrm{S}\left(\mathrm{P}_{1}\right.$, $\left.\mathrm{P}_{2}\right)=N\left(T\left(N\left(\mathrm{P}_{1}\right), N\left(\mathrm{P}_{2}\right)\right)\right)$. T-norms are basically a generalization of the usual two-valued logical conjunction [30]. The monotonicity property of t-norms ensures that the degree of truth of conjunction does not decrease if the truth values of conjuncts increase. The requirement that 1 be an identity element corresponds to the interpretation of 1 as true (and consequently 0 as false). Continuity, which is often required from the fuzzy conjunction point of view, requires that very small changes in the truth values of conjuncts should not drastically affect the truth value of their conjunction.

Some t-norm which we have used in our experiment has the parametric form:

$$
\begin{aligned}
& \text { 1. } \operatorname{Frank}(q>0): \log _{\mathrm{q}}\left(1+\frac{\left(\mathrm{q}^{\mathrm{p} 1}-1\right)\left(\mathrm{q}^{\mathrm{p} 2}-1\right)}{\mathrm{q}-1}\right) \\
& \text { 2. Hamacher: }\left(\frac{\mathrm{p}_{1} \mathrm{p}_{2}}{\left(\mathrm{q}+(1-\mathrm{q})\left(\mathrm{p}_{1}+\mathrm{p}_{2}-\left(\mathrm{p}_{1} \mathrm{p}_{2}\right)\right)\right.}\right)
\end{aligned}
$$

where $\mathrm{q}>0$ and is found to be more effective than other forms of t-norms.

\section{SCORE LEVEL FUSION BASED ON ENTROPY FUNCTION}

The Score vector $\mathrm{P}=\left[\mathrm{P}_{1}, \mathrm{P}_{2}, \ldots \ldots \mathrm{P}_{\mathrm{n}}\right]$ can be generated, if the unknown identity is to be found (with $\mathrm{N}$ biometric traits) where $\mathrm{P}_{\mathrm{i}}$ is the score generated by the ith classifier corresponding to the ith biometric trait. The Euclidean distance is thus then calculated, after feature extraction from the modalities under consideration for the purpose of delivering the scores. Based on the test image thus obtained, the Scores can be considered either to be genuine or imposter depending on the fact whether the test image belongs to the same set of training images or not respectively.

The score level fusion can be calculated by three methods. The first method being density based score level fusion where the density functions of the match score (which are usually not known), need to be calculated from a set of training scores of the classes. Density estimation can be achieved either by the parametric or non-parametric methods [31].Parametric method involves the estimation of parameters of the density from the training data. Non-parametric techniques are essentially data driven and used because they do not assume any standard form for the density function. In the classifierbased score level fusion, a pattern classifier is used to learn indirectly the relationship between the vector of scores $\mathrm{P}=\left[\mathrm{P}_{1}, \mathrm{P}_{2}, \ldots \ldots \mathrm{P}_{\mathrm{n}}\right]$ provided by the $\mathrm{N}$ matchers and the posteriori probabilities of the classes $\mathrm{P}\left(C_{i} \mid \mathrm{P}_{1}, \mathrm{P}_{2}, \ldots \ldots \mathrm{P}_{\mathrm{n}}\right)$ where $C_{i}$ is the ith class. Here, a vector of scores is treated as a feature vector that is used to categorize into one of two possible classes: imposter or genuine. Based on the training set of scores from classes, the classifier learns a decision boundary between the classes. However, the classifier is capable of obtaining the decision boundary irrespective of how the feature vectors are generated. Hence, the output scores of the different matchers can be non-homogeneous such as distance or similarity metric, different numerical ranges, etc. Also, no transformation is required prior to feeding them to a classifier. The third method for obtaining score level fusion is Combination based or Transformation-based score fusion. Practically there is a limited availability of the training data thus accurate estimation of the joint conditional densities $\mathrm{P}\left(\mathrm{P}_{1}, \mathrm{P}_{2}, \ldots \ldots \mathrm{P}_{\mathrm{n}} \mid C_{i}\right)$ for all classes is not always possible. In such situations, there is a need to directly combine the scores provided by different matchers without converting them into a posteriori probabilities. Hence, score normalization is needed to transform the scores obtained from the different classifiers into a common domain simply because of the fact combination of scores is relevant only when the scores of the individual matchers are comparable. Such normalized scores do not have any probabilistic interpretation. Consequently, in the transformed domain, the sum, max and min combination rules can be directly applied, while the product rule cannot be applied. In this paper we follow the transformation based score level fusion using Entropy Function over t-norms operators which generalize sum, max and min. For applying Entropy Function, scores $(x)$ from all the FKP's (right index, right middle, left index and left middle) must be first converted to the common domain $[0,1]$ to guarantee a meaningful combination of the scores. The normalization criterion to do this is taken as

$x^{\prime}=\frac{x-\min (x)}{\max (x)-\min (x)}$,

where $\mathrm{x}$ 'represents the normalized scores that are combined using $\mathrm{t}$-norm defined as $\mathrm{T}(x, y)=\mathrm{t}$-norm $(x, y)$. The fusion is done in an associative manner, first by combining the fusion output of the first two modalities with the third modality, and so on, until all modalities are finished. If $\mathrm{P}_{1}, \mathrm{P}_{2}, \mathrm{P}_{3} \mathrm{P}_{4}$ the normalized scores for the four FKP'S, then any two scores, say $P_{1}$ and $P_{2}$ are first combined to yield $T\left(P_{1}, P_{2}\right)$, which is in turn combined further with $\mathrm{P}_{3}, \mathrm{P}_{4}$ to yield $\mathrm{T}\left(\left(\mathrm{P}_{3}, \mathrm{P}_{4}\right), T\left(\mathrm{P}_{1}\right.\right.$, $\left.\mathrm{P}_{2}\right)$ ). The order of combination is seldom important due to the associative and commutative properties of these norms. So the combined score $\mathrm{S}$ is given by

$\mathrm{S}=\mathrm{T}\left(\left(\mathrm{P}_{3}, \mathrm{P}_{4}\right), T\left(\mathrm{P}_{1}, \mathrm{P}_{2}\right)\right)$

The fused score is checked for being genuine or imposter by comparing it with a threshold say, $\mathrm{s}$ which assigns a user as genuine if $\mathrm{S}<\mathrm{s}$, otherwise imposter.

Score level Fusion by means of Entropy Function achieves better performance as compared to the traditional methods of fusion by means of t-norms, sum and max as is visible from the results. Our proposed method employs fusion by means of Hanman \& Anirban Entropy function

$\mathrm{H}(\mathrm{p})=\sum \mathrm{pe}^{-\left(a \mathrm{p}^{3}+b \mathrm{p}^{2}+c \mathrm{p}+\mathrm{d}\right)}$

where we have kept parameters $\mathrm{a}=\mathrm{b}=\mathrm{d}=0$ and $\mathrm{c}=1$ to achieve the desired better performance.Thus reducing the above equation of Hanman \&Anirban to

$\mathrm{H}(\mathrm{p})=\sum \mathrm{pe}^{-(\mathrm{p})}$

In order to calculate and thus compare the Score level fusion by means of entropy function the parameter $(p)$ in the above reduced equation has been replaced first by the Frank t-norm

$\mathrm{t}_{\mathrm{F}}=\log _{\mathrm{q}}\left(1+\frac{\left(\mathrm{q}^{\mathrm{p} 1}-1\right)\left(\mathrm{q}^{\mathrm{p} 2}-1\right)}{\mathrm{q}-1}\right)$ giving the Modified equation of

$\mathrm{H}(\mathrm{p})=\sum \log _{\mathrm{q}}\left(1+\frac{\left(\mathrm{q}^{\mathrm{p} 1}-1\right)\left(\mathrm{q}^{\mathrm{p} 2}-1\right)}{\mathrm{q}-1}\right) \mathrm{e}^{-\log _{\mathrm{q}}\left(1+\frac{\left(\mathrm{q}^{\mathrm{p} 1}-1\right)\left(\mathrm{q}^{\mathrm{p} 2}-1\right)}{\mathrm{q}-1}\right)}$ 
and then by the Hamacher t-norm

$$
\begin{aligned}
& \mathrm{H}=\left(\frac{\mathrm{p}_{1} \mathrm{p}_{2}}{\left(\mathrm{q}+(1-\mathrm{q})\left(\mathrm{p}_{1+} \mathrm{p}_{2}-\left(\mathrm{p}_{1} \mathrm{p}_{2}\right)\right)\right.}\right) \text { giving the modified equation of } \\
& \mathrm{H}(\mathrm{p})=\sum\left(\frac{\mathrm{p}_{1} \mathrm{p}_{2}}{\left(\mathrm{q}+(1-\mathrm{q})\left(\mathrm{p}_{1+} \mathrm{p}_{2}-\left(\mathrm{p}_{1} \mathrm{p}_{2}\right)\right)\right.}\right) \mathrm{e}^{-\left(\frac{\mathrm{p}_{1} \mathrm{p}_{2}}{\left(\mathrm{q}+(1-\mathrm{q})\left(\mathrm{p}_{1}+\mathrm{p}_{2}-\left(\mathrm{p}_{1} \mathrm{p}_{2}\right)\right)\right.}\right)}
\end{aligned}
$$

in order to achieve the desired better results as compared to the corresponding fusion methods.

\section{EXPERIMENTAL RESULTS}

The simulations are performed on the PolyU's FKP database which is publicly available [32]. It consists of left index, left middle, right index, and right middle FKPs of 165 users. Each user has 12 images with each user having 48 images from four fingers. In total, the database contains 7,920 images from 660 different fingers. The database is divided into 8 training and 4 test images. We have extracted the features using Gabor Wavelets. The scores are evaluated using the Euclidean distance. We obtain $165 * 4$ genuine and $165 * 164 * 4$ impostor scores. Fig. 1 below shows the ROC of individual scores of left index, left middle, right index, and right middle. The genuine Score for Knuckle of fingers are combined using entropy function to yield the integrated genuine score, similarly the Imposter Score for knuckle of fingers are integrated using the Entropy function to yield Integrated Imposter score. The scores are then compared with the threshold of step size 0.0005 and error rates are calculated. The initial value of threshold is set to the minimum of genuine scores and the final value of threshold achieves the value of maximum of imposter scores. Finally the Error rates (False Acceptance rate (FAR) and False rejection rate (FRR)) are used to calculate the Receiver operating Characteristics (ROC) which depicts the performance of the Authentication system as compared to Frank t-norm, Hamcher t-norm, SUM and MAX. The plot Compares FAR vs. GAR (where $\mathrm{GAR}=100-\mathrm{FRR}$ ) with varying threshold values.

As is clear from the Fig.2 the Entropy based score level fusion gives better results as compared to the score level fusion of Frank t-norm, Hamcher t-norm, SUM and MAX.The Equations $11 \& 12$ are used to calculate and compare Entropy based Genuine Acceptance Rate (GAR) for different values of parameter $q$, with the corresponding Frankt-norm as depicted in Table 1 and Fig 3 and Hamacher t-norm as depicted in Table 2 and Fig. 4

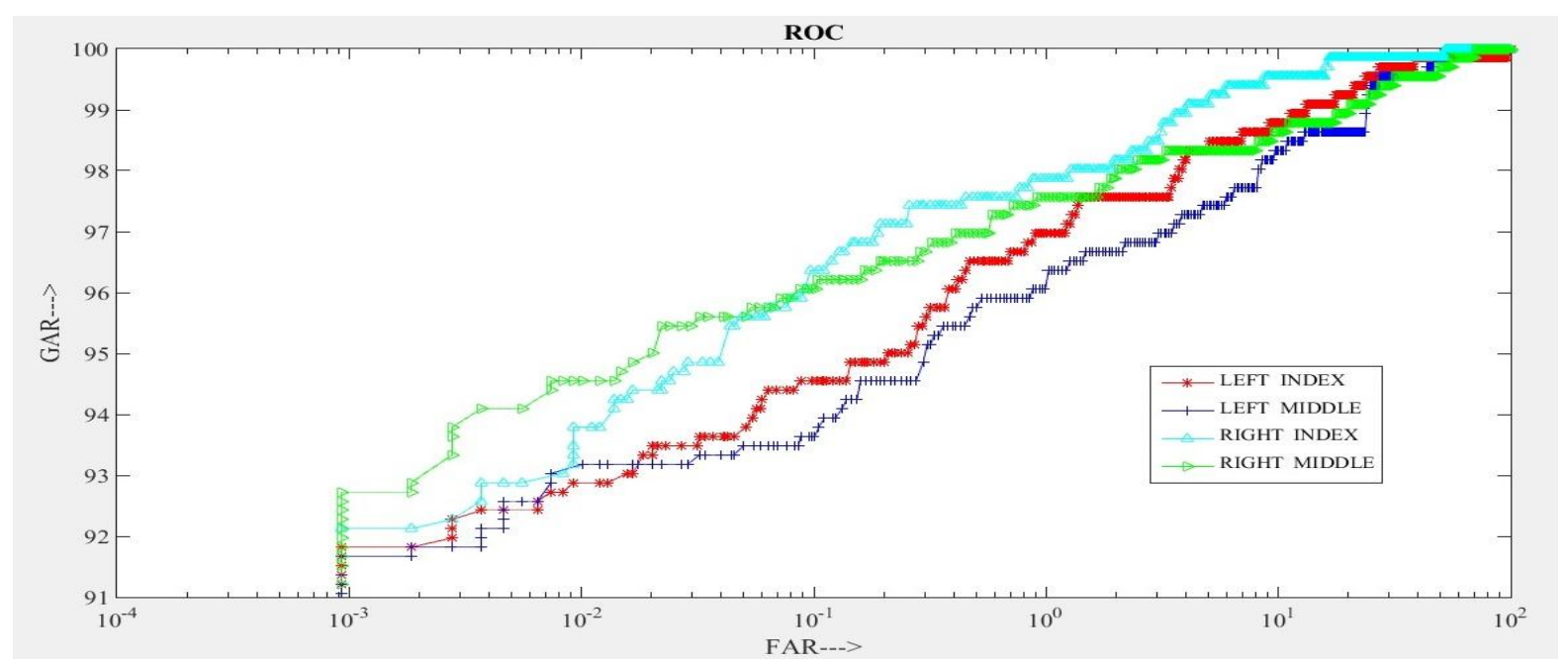

Fig 1: A comparison of ROC's of individual modality

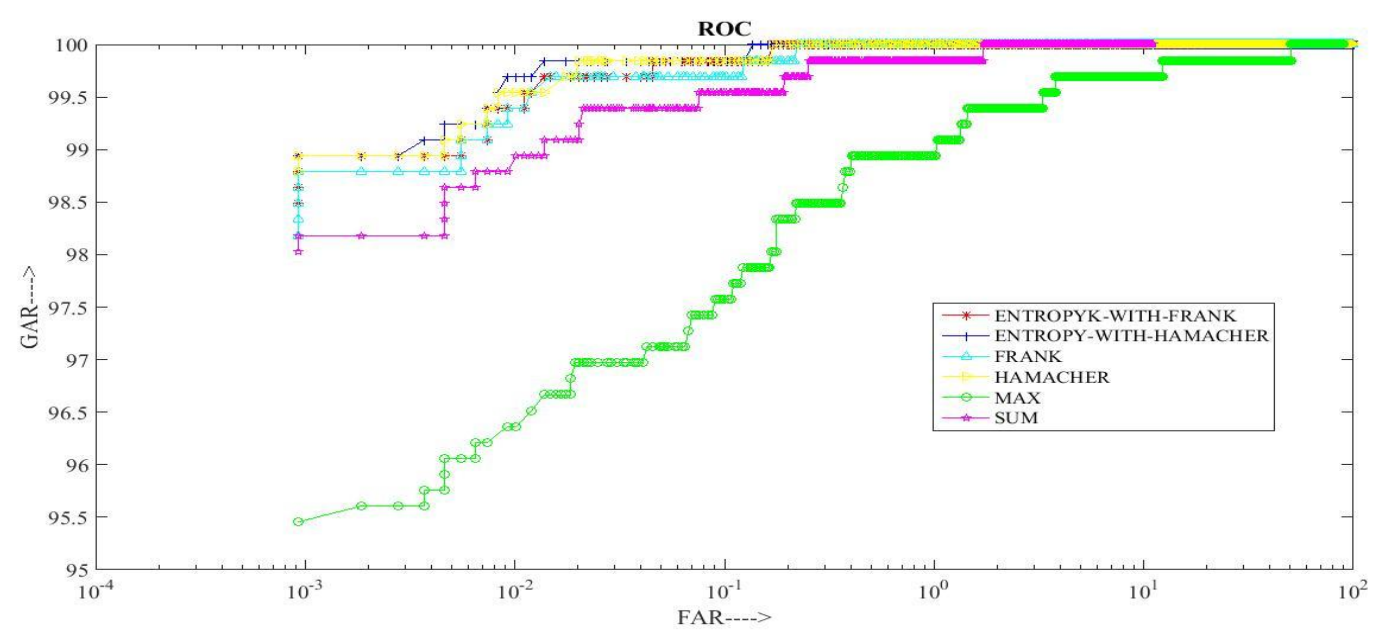

Fig 2: ROC comparing the performance of different Score level fusion methods. 


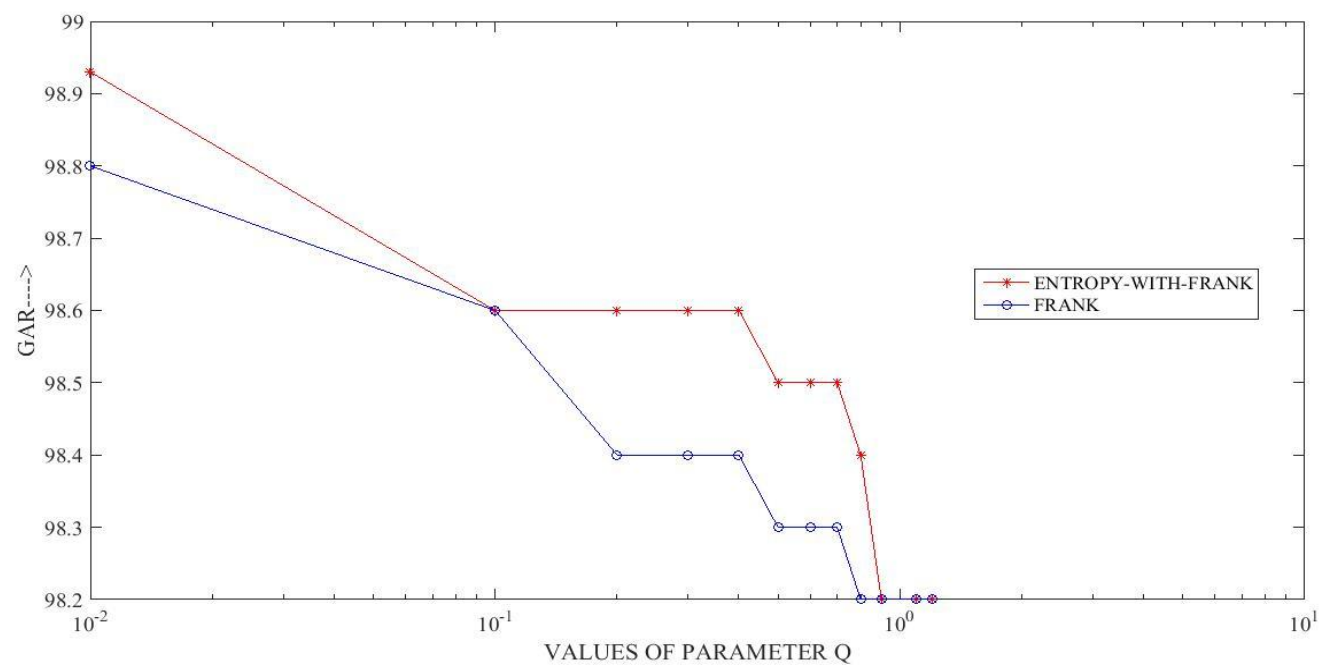

Fig 3: GAR comparison between Entropy with Frank and Frank t-norm for different values q

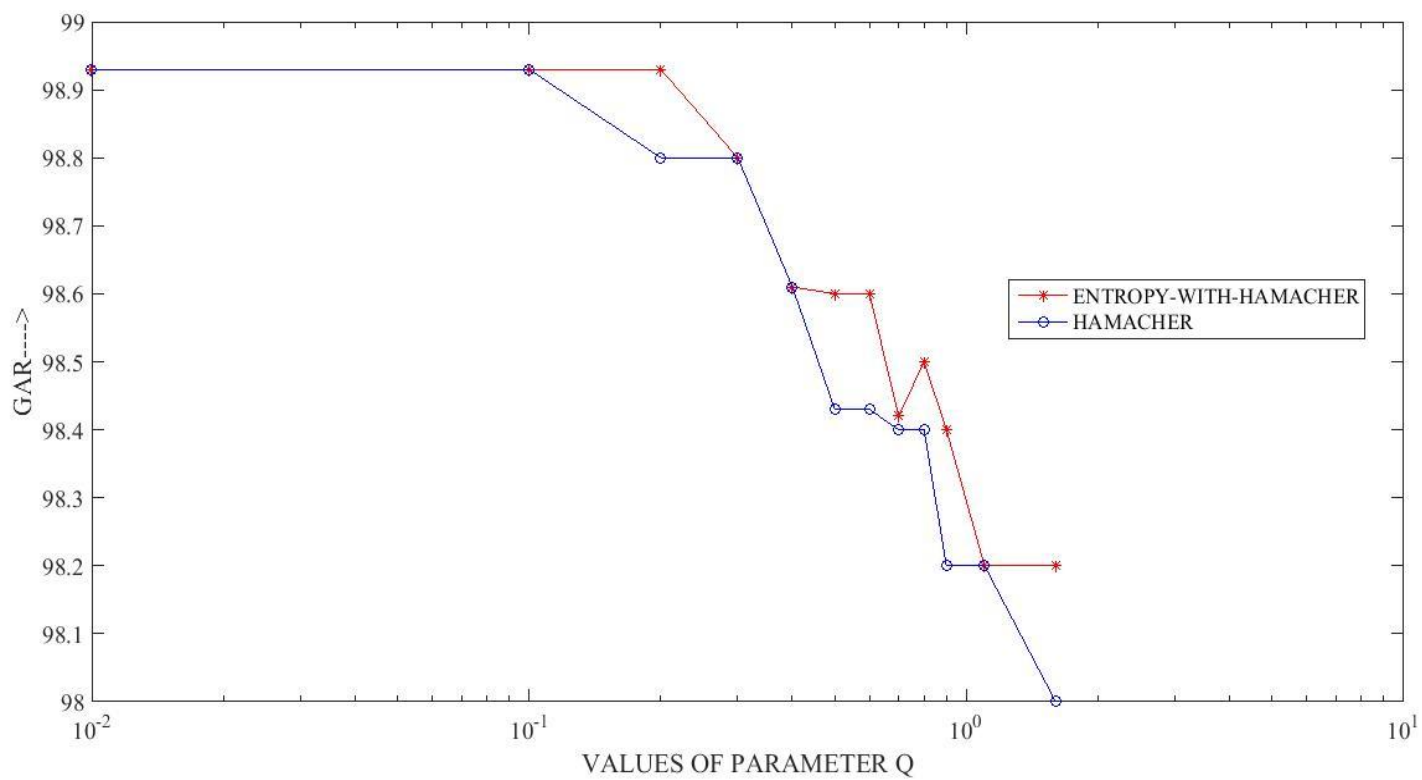

Fig 4: GAR comparison between Entropy with Hamacher and Hamacher t-norm for different values of q

Table 1. Comparison of Genuine Acceptance Rate

\begin{tabular}{|c|c|c|}
\hline Value of q & $\begin{array}{c}\text { Proposed } \\
\text { system (GAR) }\end{array}$ & $\begin{array}{c}\text { Frank t-norm } \\
\text { (GAR) }\end{array}$ \\
\hline 0.01 & 98.93 & 98.8 \\
\hline 0.2 & 98.6 & 98.4 \\
\hline 0.5 & 98.5 & 98.3 \\
\hline 0.8 & 98.4 & 98.2 \\
\hline
\end{tabular}

Table 2. Comparison of Genuine Acceptance Rate

\begin{tabular}{|c|c|c|}
\hline Value of q & $\begin{array}{c}\text { Proposed } \\
\text { system (GAR) }\end{array}$ & $\begin{array}{c}\text { Hamacher t- } \\
\text { norm (GAR) }\end{array}$ \\
\hline 0.01 & 98.93 & 98.93 \\
\hline 0.2 & 98.93 & 98.8 \\
\hline 0.5 & 98.6 & 98.43 \\
\hline 0.8 & 98.5 & 98.4 \\
\hline
\end{tabular}

\section{CONCLUSION}

In this paper, we focused on developing new effective score level fusion method for better authentication. The score level fusion combines the scores of right index, right middle, left index and left middle FKPs using the Hanman-Anirban entropy function. We have first combined the scores using the T-norms and then apply the above entropy function. Entropy based Score level fusion is tested on publicly available PolyU database and the experimental results demonstrated the efficiency and effectiveness of this new score level fusion technique. The proposed score level fusion technique can deal with any number of biometric modalities.

\section{ACKNOWLEDGMENTS}

We would like to thank our fellow students especially (Sheikh Riyaz Ul Haq \& Shiekh Burhan Ul Haque) and the faculty especially (Harsh Bhasin) for their support and valuable suggestions. 


\section{REFERENCES}

[1] A.K. Jain, A. Ross. 2004 Multibiometric systems. In Commun. ACM 47 (1) (special issue on multimodal interfaces), pages 34-40

[2] A. Jain, A. Ross and S. Prabhakar. 2003 An introduction to biometric recognition, To appear in IEEE Transaction on Circuit and System for Video Technology,

[3] Gian Luca Marcialis and Fabio Roli 2007 Serial Fusion of Fingerprint and Face Matchers, M. Haindl, MCS 2007, LNCS volume 4472, pp. 151-160, (C) SpringerVerlag Berlin Heidelberg

[4] Kartik.P, S.R. Mahadeva Prasanna and Vara.R.P. 2008 Multimodal biometric person authentication system using speech and signature features, in TENCON 2008 - 2008 IEEE Region 10 Conference, pp. 1-6.

[5] Rodriguez.L.P, Crespo.A.G, Lara.M and Mezcua.M.R. 2008 Study of Different Fusion Techniques for Multimodal Biometric Authentication, in Networking and Communications, IEEE International Conference on Wireless and Mobile Computing.

[6] J. Fierrez-Aguilar, J. Ortega-Garcia, D. Garcia-Romero, and J. Gonzalez Rodriguez. 2003 A comparative evaluation of fusion strategies for multimodal biometric verification, in Proc. 4th Int, Conf,Audio-video-based Biometric Person Authentication, J. Kittler and M. Nixon, Eds., vol. LNCS 2688, pp. 830-837.

[7] S. Viriri and R. Tapamo. 2009 Integrating Iris and Signature Traits for Personal Authentication using UserSpecific Weighting.

[8] M. Kazi and Y. Rode. 2012 multimodal biometric system using face and signature: a score level fusion approach, Advances in Computational Research, Vol. 4, No. 1.

[9] Menrit S. Fahmy, Amir F. Atyia and Raafat S. Elfouly 2008 Biometric fusion using enhanced SVM classification. In: Fourth International Conference on Intelligent Information Hiding And Multimedia Signal Processing. Harbin, pp. 1043-1048.

[10] X. Zhou, B. Bhanu. 2007 Integrating face and gait for human recognition at a distance in video. In IEEE Trans. Systems Man Cybernet, Part B: Cybernet.

[11] A. Alford, C. Hansen, G. Dozier, K. Bryant, J. Kelly, T. Abegaz, K. Ricanek, and D. L. Woodard. 2011 GECbased multi-biometric fusion, IEEE Congr. Evol. Comput., pp. 2071-2074.

[12] Slobodan Ribaric, Ivan Fratric. 2005 A Biometric Identification System Based on Eigenpalm and Eigenfinger Features, IEEE Transactions on Pattern Analysis \& Machine Intelligence, vol.27, no. 11, pp. 1698-1709.

[13] R. Snelick, U. Uludag, A. Mink, M. Indovina and A. Jain. 2005 Large Scale Evaluation of Multimodal Biometric Authentication Using State-of-the-Art Systems, IEEE Trans. Pattern Analysis and Machine Intelligence, vol. 27, no. 3, pp. 450-455, Mar.

[14] Karthik Nandakumar, Yi Chen, Sarat C. Dass, Anil Jain. 2008 Likelihood Ratio-Based Biometric Score Fusion, IEEE Transactions on Pattern Analysis \& Machine Intelligence, vol.30, no. 2, pp. 342-347.
[15] Mingxing, He, Horng, Shi-Jinn, Fan, Pingzhi, Run, RayShine, Chen, Rong-Jian, Lai, Jui-Lin, Muhammad, Khurram Khan, Kevin, Octavius Sentosa, 2010. Performance evaluation of score level fusion in multimodal biometric systems. Pattern Recognit. 43 (5) $1789-1800$

[16] L. Nanni, A. Lumini, S. Brahnam. 2011 Likelihood ratio based features for a trained biometric score fusion. Expert Systems Appl.: Int. J. 38 (1), 58-6.

[17] Kar-Ann Toh, Jaihie Kim, Sangyoun Lee 2008. Biometric scores fusion based on total error rate minimization. Pattern Recognit. 41 (3), 1066-1082.

[18] Hyun-Ae Park, Kang Ryoung park 2007. Iris recognition based on score level fusion by using SVM. Pattern Recognition Lett 28 (15), 2019-2028.

[19] J. Kittler, M. Hatel, R. P. W. Duin, and J. Matas. 1998 On combining classifiers. IEEE Transactions on Pattern Analysis and Machine Intelligence, 20(3):226-239.

[20] Ajay Kumar and Vivek Kanhangad. 2010 A New Framework for Adaptive Multimodal Biometric Management, IEEE Transactions on Information Forensic and Security, Val 5,No-I.

[21] Madasu Hanmandlu, Jyotsana Grover, Ankit Gureja , H M. Gupta. 2011 Score level fusion of multimodal biometrics using triangular norms, Pattern Recognition Letters, v.32 n.14, p.1843-1850, October.

[22] Shannon, C. E. (1948). A mathematical theory of communication. Bell System Technical Journal, 27, 379423 .

[23] A. Renyi, 1970. Probability Theory. North-Holland, Amsterdam.

[24] C. Tsallis, S. Abe, Y. Okamoto, 2001. (Lecture notes in Physics). Nonextensive statistical mechanics and its applications, vol. 560. Springer, Berlin, New York.

[25] Pal, N. R., \& Pal, S. K. 1992 Some properties of the exponential entropy. Information Sciences, 66(1-2), 113-117.

[26] M. Hanmandlu, \& A. Das. 2011 Content-based image retrieval by information theoretic measure. Defence Science Journal, 61(5), 415-430.

[27] Mamta; M. Hanmandlu. 2015 Multimodal Biometric System Built on the New Entropy Function for Feature Extraction and the Refined Scores as a Classifier. Expert Syst. Appl., 42, 3702-3723.

[28] Bonissone, P.P., 1987. Summarizing and propagating uncertain information with triangular norm. Int. J Approximate Reasoning 1 (1), 71-101.

[29] S. Weber, 1983. A general concept of fuzzy connectives, negations and implications based on t-norms and $\mathrm{t}$ conorms. Fuzzy Sets Systems 11, 115-134.

[30] Duda, R.O., Hart, P.E., Stork, D.G., 2001. Pattern Classification. John Wiley \& Sons, New York.

[31] PolyU FKP Database [Online]. http://www4.comp.polyu.edu.hk/ biometrics/FKP.htm 